\title{
Development and Implementation of a Supervisor Strategy and Sliding Mode Control Setup for Fuel Cell-Based Hybrid Generation Systems
}

\author{
J.J. Moré*†, P.F. Puleston*‡, C. Kunusch** and M. Allue**
}

\begin{abstract}
This paper presents the development and experimental results of a supervisor strategy and a sliding mode control setup to improve the performance of hybrid generation systems. The topology in this work is conformed by a core, comprising a Fuel Cell Module and a Supercapacitor Module, in combination with an Alternative Energy Source Module and an electrolyzer. In particular, a wind power turbine is considered as alternative power source, to attain a hybrid generation system fully relying on renewable energy. Firstly, a supervisor strategy is proposed to manage the power flows of the subsystems and coordinate the system as a whole. Subsequently, a sliding mode control setup for combined operation of the DC/DC power converters of the Fuel Cell/Supercapacitor core is presented to track the power references synthesized by the supervisor control. Both control levels, supervisor strategy and sliding mode controllers, are implemented and assessed through extensive experimental tests, under different wind conditions and heavy load changes.
\end{abstract}

Index Terms-Hybrid Systems, Fuel cells, Wind power generation, Supercapacitors, Sliding Mode Control, Supervisor Control

\section{INTRODUCTION}

In the last few years, hybrid generation systems (HGS) have become an important research field all over the world. In particular, renewable energy hybrid systems are of special interest, thrusted by the increasing environmental awareness and fossil fuels depletion. In this area, fuel cell-based systems emerge as an excellent choice due to its high efficiency and long term operation [1], [2], [3]. Among them, those involving PEM fuel cells have been extensively studied in both mobile [4], [5], [6] and stationary applications [7], [8], [9], [10].

One of the key issues when dealing with PEM fuel cellbased hybrid systems, is to avoid fast changes of the stack current, to prevent irreversible damage to the membranes. To this end, a fast response power module must be integrated into the HGS. In particular, supercapacitors based power modules emerge as an efficient solution [11], [12], [13].

In order to accomplish a generation system fully relying on renewable energy, a sustainable hydrogen production approach is required. In this sense, the incorporation of a wind energy conversion systems (WECS) and an electrolyzer has demonstrated to be a versatile combination [14]. This topology allows to store the wind energy surplus to use it when it is required (see a block schematic in Fig. 1). Such hybrid system, involving manifold sinks and sources, must be

* CONICET and LEICI, Facultad de Ingeniería, Universidad Nacional de La Plata, Argentina

†jmore@ing.unlp.edu. ar

\$Marie Curie IIF Fellow at IRII (CSIC-UPC), Barcelona, Spain

** Institut de Robòtica i Informàtica industrial (CSIC-UPC), Parc Tecnològic de Barcelona. C/ Llorens i Artigas 4-6. 08028 Barcelona, Spain comprehensively controlled and supervised to ensure a proper operation of the overall system and its constituent subsystems. The supervisor strategy and dedicated sliding mode controllers (SMC) for a fuel cell-based HGS developed in this paper proved to be a proficient solution for such task.

The article is organized as follows. Section II presents the complete system and subsystems descriptions. In Section III the supervisor strategy is proposed and the sliding mode controllers of the DC/DC converters are designed. In Section IV, the controllers implementation and experimental results under different operating conditions are presented. Finally, in Section V, conclusions are given and discussed.

\section{SYSTEM DESCRIPTION}

\section{A. Hybrid Generation System Topology}

This subsection introduces a general description of the hybrid generation system topology under study. The core of the system comprises a Fuel Cell Generation Module (FCGM) and a Supercapacitor Bank Module (SCBM). The former is based on a PEM fuel cell stack fed with pure hydrogen and air.

The FCGM is connected to a DC bus through a unidirectional DC/DC boost converter, in order to adapt the different voltage values (see an schematic of the complete system in Fig. 1). The SCBM is based on a supercapacitors bank, connetected to the above mentioned DC bus through a bidirectional DC/DC

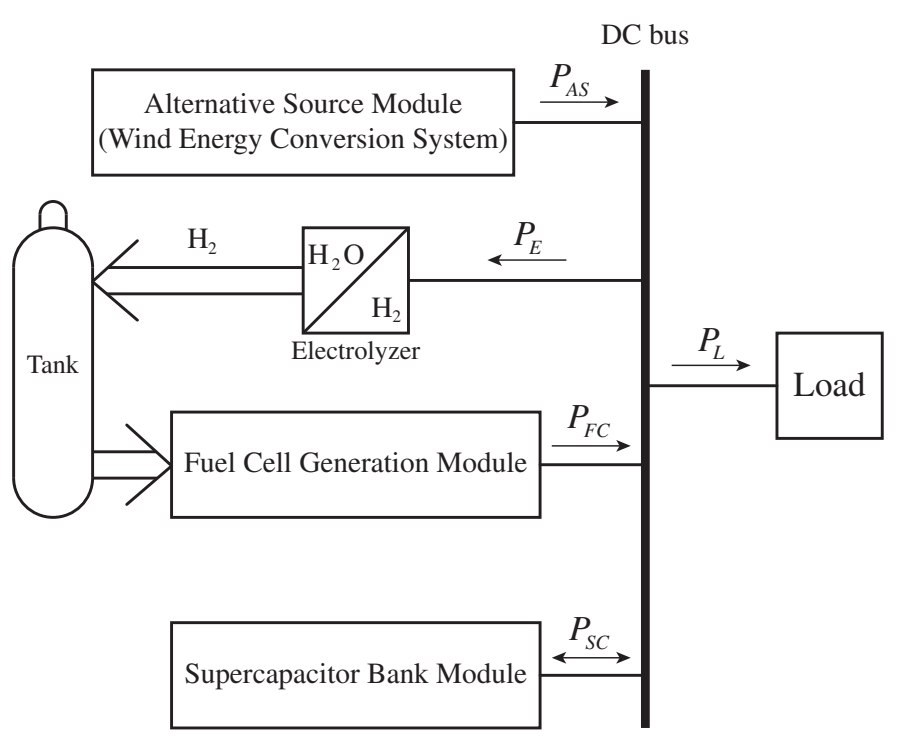

Fig. 1. Hybrid System Block Diagram 
converter. This power converter allows the supercapacitors to deliver (discharge mode) or absorb (charge mode) energy.

Different alternative sources can be connected to the DC bus in this hybrid topology. In particular, a Wind Energy Conversion System is considered in this work. The alternative energy source, attempts to satisfy the total power demand (i.e., load and supplementary devices of the system). If it is not sufficient or the demand varies exceedingly fast, then active action must be taken by the FCGM-SCBM core, to deliver the required extra power.

In order to complete the hybrid system, an electrolyzer is connected to the DC bus. The Electrolyzer System (ES) is intended to produce the hydrogen to feed the FCGM tank, when excess power is available from the alternative source. It is important to remark that the ES includes all the subsystems needed to produce, compress and store the hydrogen into the tank. Also note that without loss of generality the load is considered to be directly connected to the DC bus. Nonetheless, $\mathrm{AC}$ loads can be contemplated as well by including a DC/AC power converter.

\section{B. Hybrid Test Station Technical Description}

The actual HGS used in this paper is a versatile hybrid test station specially developed for control design and experimental evaluation in the Fuel Cell Laboratory at the Institut de Robótica i Informàtica industrial (CSIC-UPC). The FCGM is based on a Nexa ${ }^{\circledR}$ fuel cell generation system from Ballard ${ }^{\circledR}$. This stack is capable to deliver up to $1.2 \mathrm{~kW}$ to a DC load with unregulated output voltage. The maximum rated power is obtained when the output current reaches $46 A$ at a nominal output voltage of $26 \mathrm{~V}$. The open circuit voltage under regular conditions is about $48 \mathrm{~V}$. To prevent that the switched current of the converter affects the Fuel Cell operation, a low-pass filter with a cutoff frequency of $500 \mathrm{~Hz}$ is set between the Nexa ${ }^{\circledR}$ and de DC/DC power converter.

The SCBM is based on a Maxwell ${ }^{\circledR} 165 \mathrm{~F}$ supercapacitors bank, with a rated voltage of $48 \mathrm{~V}$ and a rated constant current of $98 A$. Both DC/DC power converters are built using two columns of IGBT's of a Semikron ${ }^{\circledR}$ three-phase inverter. The maximum switching frequency is $20 \mathrm{kHz}$, the maximum voltage is $400 \mathrm{~V}$ and the maximum mean current is $75 \mathrm{~A}$. The inductor of each converter is of $35 \mu H y$ and the DC bus capacitance is $2720 \mu \mathrm{F}$.

A programmable power source is connected to the DC bus to emulate different Alternative Source Modules of the test bench. It is a NL Source-Sink of Höcherl \& Hackl GmbH ${ }^{\circledR}$ The maximum voltage is $80 \mathrm{~V}$ and it can deliver up to $3.2 \mathrm{~kW}$. In this paper, the programmable source is used to emulate the Wind Energy Conversion System, as indicated in the previous section.

The test bench also has a programmable ZL Electronic DC load, also of Höcherl \& Hackl $\mathrm{GmbH}^{\circledR}$, that can reach $80 \mathrm{~V}$ and a maximum constant power of $3.4 \mathrm{~kW}$ and a peek power of $3.4 \mathrm{~kW}$. It represents the system external load and currently, it is also in charge of emulating the variable power demand of the ES.

\section{CONTROL DESIGN}

This section presents the two-levels control setup developed for the HGS. The upper level of the control hierarchy is a supervisor control strategy that globally handles the HGS power flows. The lower level corresponds to dedicated sliding mode controllers for the FCGM-SCBM subsystems.

\section{A. Upper Level: Supervisor Control Strategy:}

The main objective of the HGS is to adequately satisfy the external load demand. Additionally, provided that spare power is available from the alternative energy source, secondary objectives are to maintain the SCBM charged at a desired voltage and to generate hydrogen if the tank is not full. Then, the following supervisor control strategy is proposed to calculate the coordinated power references for each module of the HGS (the schematic flow diagram in Fig. 2 helps the understanding of the subsequent explanation. The power names used in the flow chart are itemized in Table I).

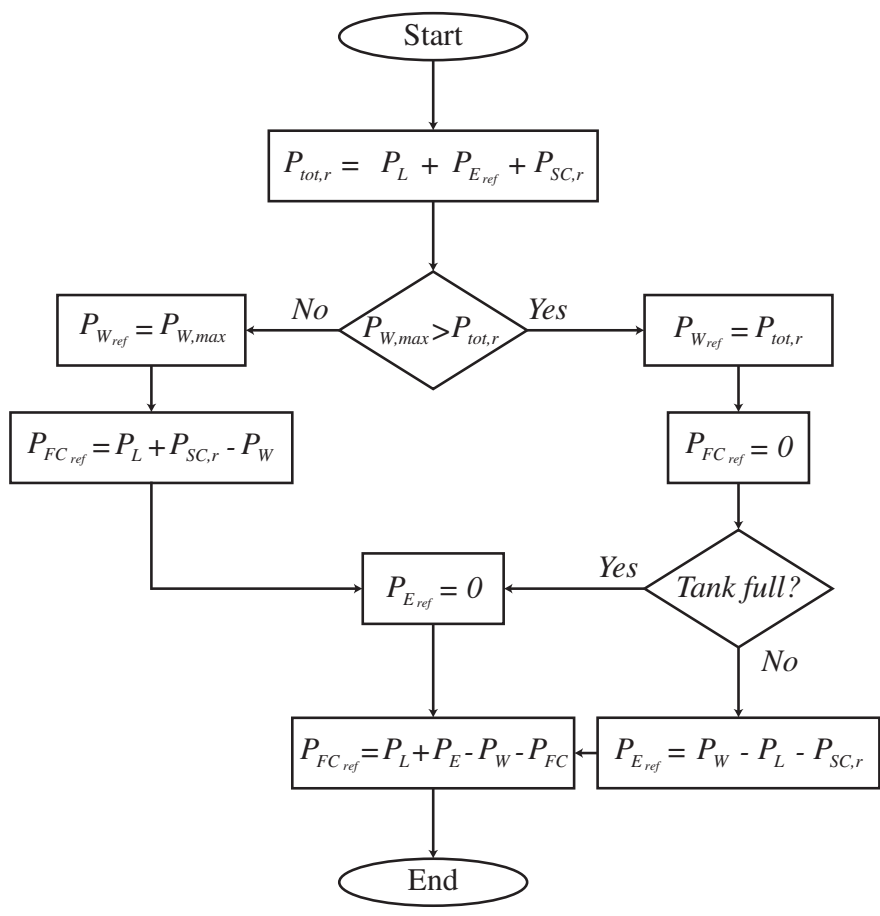

Fig. 2. Supervisor Strategy Flow Diagram

\begin{tabular}{|l|l|}
\hline$P_{t o t, r}$ & Total power demand to the DC bus \\
$P_{L}$ & External load demand \\
$P_{E_{r e f}}$ & ES power reference \\
$P_{S C, r}$ & Power required to recharge the SCBM \\
$P_{W, \max }$ & Actual maximum available power \\
$P_{W_{\text {ref }}}$ & WECS power reference \\
$P_{F C}$ & FCGM power reference \\
$P_{W}$ & WECS power \\
$P_{E}$ & ES power \\
$P_{F C}$ & FCGM power \\
\hline
\end{tabular}

TABLE I

POWER NAMES IN THE FLOW DIAGRAM OF FIG. 2 
Firstly, the WECS power reference must be obtained. To this end, the total power demand to the DC bus is computed:

$$
P_{t o t, r}=P_{L}+P_{E_{r e f}}+P_{S C, r},
$$

where $P_{L}$ is the external load demand, $P_{E_{\text {ref }}}$ is the ES power reference and $P_{S C, r}$ is the power required to recharge (or discharge) the SCBM, depending on the desired supercapacitors voltage value. Note that, in accordance with Fig. 1, all the powers are referred to the DC bus side.

If the available power of the wind energy module is higher than $P_{t o t, r}$, then the WECS must satisfy the total demand $P_{t o t, r}$. Conversely, when the available wind power is lower than $P_{t o t, r}$, the WECS should deliver its maximum available power, therefore:

$$
P_{W_{r e f}}=\left\{\begin{array}{ll}
P_{W, \max } & P_{W, \max }<P_{t o t, r} \\
P_{t o t, r} & P_{W, \max } \geq P_{t o t, r}
\end{array},\right.
$$

where $P_{W_{\text {ref }}}$ is the WECS power reference and $P_{W, \max }$ is the actual maximum available power, function of the wind speed.

Regarding the ES, its power reference is determined considering the amount of hydrogen in the tank and the availability of extra wind power. In other words, if the tank is full, the electrolyzer must not produce any hydrogen. Conversely, when the tank is not full, the ES reference $P_{E_{r e f}}$ should be equal to the excess of wind power, limited to the maximum admisible power. So, considering the wind power balance:

$$
P_{W, b a l}=P_{W}-P_{L}-P_{S C, r}
$$

where $P_{W}$ is the actual WECS power delivered to de DC bus, $\mathrm{Vol}_{H_{2}}$ and $\mathrm{Vol}_{H_{2}, \max }$ are the actual and maximum hydrogen volume in the tank respectively, the ES power reference can be posed:

$$
P_{E_{\text {ref }}}=\left\{\begin{array}{lc}
0 & \operatorname{Vol}_{\mathrm{H}_{2}} \geq \text { Vol }_{\mathrm{H}_{2}, \text { max }} \text { or } P_{W, \text { bal }}<0 \\
P_{W, \text { bal }} & 0 \leq P_{W, \text { bal }} \leq P_{E, \max } \\
P_{E, \text { max }} & \text { else }
\end{array}\right.
$$

where $P_{E, \max }$ is the maximum power that can be processed by the ES.

The computation of the SCBM charge/discharge power $P_{S C, r}$ is based on the supercapacitors bank voltage:

$$
P_{S C, r}=K_{c h, p} \cdot\left(V_{S C_{r e f}}-v_{S C}\right)+z_{e},
$$

where:

$$
\dot{z}_{e}=K_{c h, i} \cdot\left(V_{S C_{r e f}}-v_{S C}\right),
$$

and $V_{S C_{\text {ref }}}$ is the supercapacitors voltage reference, $v_{S C}$ the supercapacitors voltage, and $K_{c h, p}$ and $K_{c h, i}$ are gains that should be designed to obtain the desired charge/discharge dynamics, in order to avoid overloading of the power sources. For instance, an extremely high value of $K_{c h, p}$ would result in a rapid charge of the supercapacitors bank, but at the expense of producing high power demand to the sources.

Next, the FCGM power reference to be used in the subsequent SMC is computed taking into account the load power demand that cannot be satisfied by the WECS. It is important to stress that while the FCGM is delivering power, the ES must not be operative, in order to maximize the overall efficiency of the whole system. So, the FCGM reference results:

$$
P_{F C_{r e f}}=\left\{\begin{array}{lc}
-P_{W, b a l} & P_{W, b a l}<0 \\
0 & \text { else }
\end{array} .\right.
$$

Finally, the SCBM power reference required for the sliding surface in the following subsection is obtained from the power balance in the DC bus:

$$
P_{S C_{\text {ref }}}=P_{L}+P_{E}-P_{W}-P_{F C},
$$

where $P_{E}, P_{W}$ and $P_{F C}$ are the ES, WECS and FCGM power respectively.

Note that in stationary state (i.e. when all modules have reached their references power values) the power reference equals the charge/discharge power 5 :

$$
P_{S C_{r e f}}=P_{S C, r}
$$

\section{B. Lower Level: FCGM/SCBM Core Sliding Mode Control}

The previous section presented the supervisor control strategy of the complete hybrid system that computes the power references for the different modules. To track those references, in this section, a sliding mode robust control set up is developed to command the power converters of the FCGM and the SCBM. In Fig. 3, a schematic circuit diagram of the considered converters and subsystems is presented.

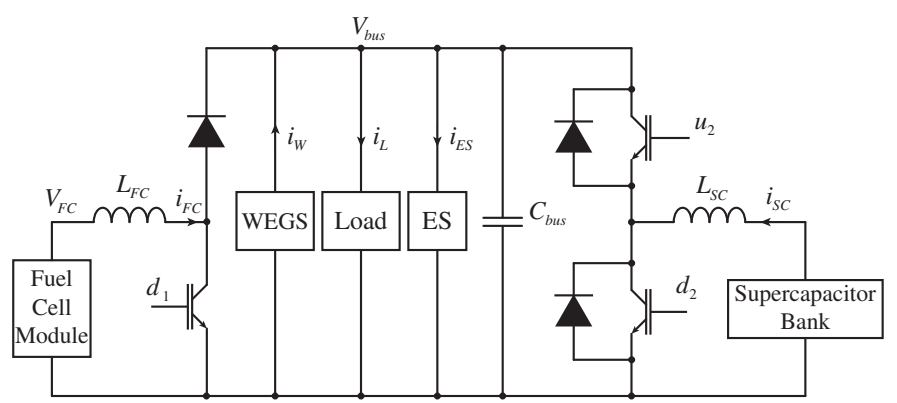

Fig. 3. Hybrid System Circuit Diagram

Equations (10) to (13) represent the average model of the system dynamics, considering losses in the converter inductors:

$$
\begin{aligned}
& \dot{i}_{F C}=\frac{v_{F C}}{L_{F C}}-\frac{R_{L f c} \cdot i_{F C}}{L_{F C}}-\frac{v_{b u s}}{L_{F C}} \cdot u_{1} \\
& \dot{i}_{S C}=\frac{v_{S C}}{L_{S C}}-\frac{R_{L s c} \cdot i_{S C}}{L_{S C}}-\frac{v_{b u s}}{L_{S C}} \cdot u_{2} \\
& \dot{v}_{b u s}=-\frac{i_{0}}{C_{b u s}}+\frac{i_{F C}}{C_{b u s}} \cdot u_{1}+\frac{i_{S C}}{C_{b u s}} \cdot u_{2} \\
& \dot{v}_{S C}=-\frac{i_{S C}}{C_{S C}}
\end{aligned}
$$

where:

$$
\begin{aligned}
v_{F C}= & N_{F C} \cdot\left(E_{o c}-R_{F C} \cdot i_{F C}-\right. \\
& \left.-A \cdot \log \left(i_{F C}\right)-m \cdot \exp \left(n \cdot i_{F C}\right)\right)
\end{aligned}
$$

is the output voltage of the FC as a function of $i_{F C}$, and: 
$i_{F C}, i_{S C}$ the FCGM and SCBM current;

$v_{b u s}, v_{S C}$ the DC bus and SCBM voltage;

$C_{\text {bus }}, C_{S C}$ the DC bus and SCBM capacitance;

$L_{F C}, L_{S C}$ the FCGM and SCBM converter inductances;

$i_{0} \quad$ the lumped current value of load, ES and WECS subsystems, i.e.:

$$
i_{0}=i_{L}+i_{E S}-i_{W}
$$

$u_{i}=1-d_{i}$ the duty cycle of the control signal $i$. Note that $d_{i} \in[0,1]$;

$R_{L f c} \quad$ the FC converter inductor resistance;

$R_{L s c} \quad$ the SCBM converter inductor resistance;

$N_{F C} \quad$ the number of cells of the FC stack;

$E_{o c} \quad$ the open loop voltage of a cell;

$R_{F C} \quad$ the linear resistance of the FC;

$A \quad$ the slope of the Tafel equation;

$m, n \quad$ constants of the mass transfer overvoltage equation.

Then, the sliding surfaces of both power converters are designed, jointly considering the main objectives of the FCGM and SCBM, as follows. The control algorithm must guarantee a constant DC bus voltage and ensure that the FCGM generated power tracks the reference computed by the supervisor control, even under heavy load variations. The algorithm must also take into account not only the value, but also the admisible rate of change of the FCGM current. The latter should be bounded to protect the device and extend its durability, given that fast current changes may produce thermal stress at the catalyst surface, decreasing the membrane lifetime.

In this way, the SCBM must complement the FCGM and the WECS powers to adequately supply the load during fast transient conditions. This requires that the SCBM converter acts rapidly to reject the effect of abrupt load variations. After the transient, the FCGM or the WECS must slowly recharge the SCBM to a desired value.

Then, to design the FCGM/SCBM control setup in the sliding mode framework, the aforementioned objectives and restrictions are embedded in two complementary sliding surfaces, based on the power references supplied by the supervisor control level.

1) FCGM Converter Sliding Surface:

The surface is designed to follow smooth power variations, considering the FC current limitations mentioned above. In stationary state, the FCGM must supply the power reference given by the supervisor control or its maximum nominal value, hence the proposed sliding variable is:

$$
s_{1}=i_{F C}-\tilde{I}_{F C_{r e f}}
$$

where $\tilde{I}_{F C_{r e f}}$ is the rate limited FCGM current reference, that is, the current that the FCGM has to deliver to supply the power reference $P_{F C_{r e f}}$ (from (7)):

$$
I_{F C_{r e f}}=\frac{P_{F C_{r e f}}}{v_{F C}},
$$

with a current slew rate limit set according to the maximum rate of change allowed by the fuel cell system.
2) Supercapacitors Converter Sliding Surface:

The SCBM converter sliding surface must take into account the FCGM current reference rate limitation and the slow response of the WECS to track load power variations. This means that the SCBM has to deal with the high frequency load changes and ensure the power balance in the DC bus. These goals can be attained by setting DC bus voltage regulation as the control objective for the SCBM converter. To this end, the sliding variable is designed with the DC bus voltage error $\left(v_{\text {bus }}-V_{\text {bus }_{\text {ref }}}\right)$ as first term. In addition, a current error term is included in the surface to obtain a stable dynamics for the converter, avoiding non-minimum phase behaviour due to direct voltage regulation/tracking [15]:

$$
s_{2}=\left(v_{b u s}-V_{b u s_{r e f}}\right)+k_{b u s} \cdot\left(i_{S C}-\tilde{I}_{S C_{r e f}}\right),
$$

where:

$$
\begin{aligned}
& V_{\text {bus }} \text { ref } \\
& k_{\text {bus }} \\
& \tilde{I}_{S C_{\text {ref }}}
\end{aligned}
$$$$
k_{\text {bus }} \quad \text { is a positive surface gain selected to obtain a }
$$
desired close loop $v_{\text {bus }}$ dynamics. is the SCBM current reference, calculated as:

$$
\tilde{I}_{S C_{r e f}}=\frac{P_{S C_{r e f}}^{*}}{v_{S C}}
$$

where $P_{S C_{r e f}}^{*}$ is the SCBM power reference computed in (8), referred to the supercapacitor side of the converter.

\section{3) Computation of the Control Action:}

The first step in the design of the control actions is the computation of the equivalent control, i.e. the continuous control action required to maintain the system confined in the sliding surface $s_{i}=0 ; i=1,2$ [16].

Assuming a sliding mode switching strategy:

$$
u_{i}=\left\{\begin{array}{cc}
U_{i}^{+}(\cdot) & s_{i}>0 \\
U_{i}^{-}(\cdot) & s_{i}<0
\end{array} ; i=1,2\right.
$$

where $U_{i}^{+}(\cdot)$ and $U_{i}^{-}(\cdot)$ are continuous functions, the equivalent controls can be obtained by solving the equations $\dot{s}_{i}=0$ for $u_{i}$, with $s_{i}=0$ [16].

For the case of the FCGM converter, neglecting the losses in the converters inductors, results:

$$
\begin{aligned}
\dot{s}_{1} & =\dot{i}_{F C}-\dot{I}_{F C, r e f} \\
& =\frac{v_{F C}}{L_{F C}}-\frac{v_{\text {bus }}}{L_{F C}} \cdot u_{1}-\dot{I}_{F C, r e f}=0,
\end{aligned}
$$

and consequently:

$$
u_{1, e q}=\frac{v_{F C}-L_{F C} \cdot \dot{I}_{F C, r e f}}{v_{b u s}}
$$

In the case of the SCBM converter, taking:

$$
\dot{s}_{2}=\dot{v}_{b u s}+k_{b u s} \cdot\left(\dot{i}_{S C}-\dot{\tilde{I}}_{S C_{r e f}}\right)=0
$$


and solving for $u_{2}$, with $s_{2}=0$, results:

$$
\begin{aligned}
u_{2, e q}= & \frac{\frac{i_{F C}}{C_{b u s}} \cdot u_{1, e q}-k_{b u s} \cdot \dot{\tilde{I}}_{S C, r e f}}{\left(\frac{k_{b u s} \cdot v_{b u s}}{L_{S C}}-\frac{i_{S C}}{C_{b u s}}\right)}+ \\
& +\frac{\frac{k_{b u s} \cdot v_{S C}}{L_{S C}}-\frac{i_{0}}{C_{b u s}}}{\left(\frac{k_{b u s} \cdot v_{b u s}}{L_{S C}}-\frac{i_{S C}}{C_{b u s}}\right)},
\end{aligned}
$$

where:

$$
i_{S C}=\frac{V_{\text {bus }_{\text {ref }}}}{k_{b u s}}-\frac{v_{b u s}}{k_{b u s}}+\tilde{I}_{S C_{r e f}}
$$

Then, the SMC for the converters are proposed as follows:

$$
u_{i}=u_{i, e q}+M_{i} \cdot \operatorname{sign}\left(s_{i}\right),
$$

for $i=1,2$, with $M_{i} \in[0,1]$ the discontinuous actions constants. These two-terms control actions allows to reduce the chattering effect due to actual non-ideal operation, by steering the system to the neighborhood of $s_{i}=0$ with the continuous term $u_{i, e q}$ and, hence, requiring a smaller discontinuous action to ensure the existence of a robust sliding mode regime. Particularly, in actual systems such as this application, $M_{i}$ are empirically tuned through experimental tests, aiming to attain the maximum values, compatible with admissible chattering requirements in real operation.

\section{4) Implementation Issues:}

The implementation of the proposed controllers in real HGSs entails addressing several practical issues, depending on the actual limitations of the available hardware/software set up.

In particular, for the experimental test station described in subsection II-B, the calculations of the continuous control terms $u_{i, e q}$ represent an exacting computational effort. Therefore simple approximations, suitable for real-time operations, can be obtained by evaluating (22) and (24) for the references values, assuming negligible time derivatives and using equations (8) and (15) for the latter. It yields the following simplified continuous terms for implementation of the SM control law (26):

$$
\begin{aligned}
u_{1, \text { cont }} & =\frac{v_{F C}}{v_{\text {bus }}}, \\
u_{2, \text { cont }} & =\frac{v_{S C}}{v_{\text {bus }}},
\end{aligned}
$$

turning theoretical (26) into implementable:

$$
u_{i}=u_{i, \text { cont }}+M_{i} \cdot \operatorname{sign}\left(s_{i}\right),
$$

for $i=1,2$.

Likewise, referring $P_{S C_{r e f}}$ to the supercapacitors side considering the converter losses $\left(P_{S C_{r e f}}^{*}\right.$ in (19)) can also be a time-consuming process and, additionally, a source of error in the reference, due to model uncertainties. Then, to overcome these issues, the following expression is proposed to compute the SCBM current reference:

$$
I_{S C_{r e f}}=\frac{P_{S C_{r e f}}}{v_{S C}}+z_{I_{S C}}
$$

where the first term corresponds to the ideal current reference for the SCBM, directly obtained from the SCBM power reference (8). The second one is an integral term, intended to compensate the error introduced by the unmodeled converter losses in the model:

$$
\dot{z}_{I_{S C}}=k_{i} \cdot\left(v_{\text {bus }}-V_{\text {bus }, \text { ref }}\right)
$$

where $k_{i}$ is the integral term gain.

It is also important to note that, for practical simplicity, $M_{i}$ are constants but they can be functions of $i_{F C}$ and $i_{S C}$, respectively, to better take into account neglected resistive losses and wide-range operating conditions.

5) Discontinuous Conduction FCGM Control Implementation:

The bidirectional SCBM converter always operates in continuous conduction mode. However, under certain conditions, the FCGM converter may enter periods of discontinuous operation. In those cases, the FCGM current becomes algebraically dependent on the system states and the duty cycle $u_{1}$, as follows:

$$
i_{F C}=\frac{T_{s}}{2 L_{F C}} \cdot \frac{v_{F C} \cdot v_{\text {bus }}}{\left(v_{\text {bus }}-v_{F C}\right)} \cdot\left(1-u_{1}\right)^{2},
$$

where $T_{s}$ is the switching period of the PWM control signal.

This algebraic relation means that during those periods an order reduction occurs in the system (10)-(13) hence, theoretically, the FCGM sliding surface and control law should be redefined. However in practice, to avoid complex control structure switching and to reduce the overall computational cost, the control structure (29) can be maintained but with a different continuous term. Instead of (27), in discontinuous conduction mode, the following continuous control term should be used:

$$
u_{1, \text { cont }}=1-\sqrt{\frac{2 L_{F C}}{T_{s}} \cdot \frac{\left(v_{b u s}-v_{F C}\right)}{v_{F C} \cdot v_{b u s}} \cdot I_{F C_{r e f}}} .
$$

obtained from (32) evaluated for $i_{F C}=I_{F C_{\text {ref }}}$.

This equation is valid for $i_{F C}$ operating below the critical current $I_{F C, \text { crit }}$ :

$$
I_{F C, \text { crit }}=\frac{T_{s}}{2 L_{F C}} \cdot v_{F C} \cdot\left(1-u_{1}\right) .
$$

which is the limit current between continuous and discontinuous conduction modes of the FCGM.

The calculation of $u_{1, \text { cont }}$ with equation (33) demands a high on-line processing effort. So, for implementation in actual systems such as the available experimental station, it is important to reduce this computational load. To this end, an approximation through a two segment piecewise linear function is utilized. Fig. 4 shows an example of both curves for the whole current range, including continuous conduction mode.

\section{EXPERIMENTAL RESULTS}

This section presents the experimental results of the proposed HGS control setup, operating under variable wind regime and exacting power demand. The control strategy was firstly assessed through simulations considering up to $10 \%$ 


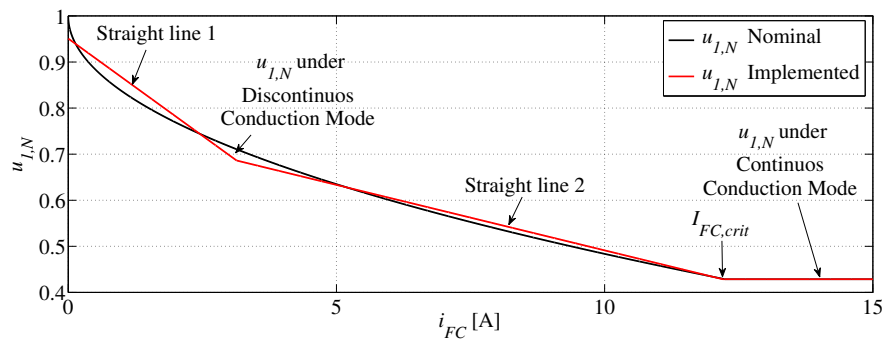

Fig. 4. Nominal control action $u_{1, \text { cont }}$

model parameters uncertainty. The simulation results were highly satisfactory hence both, the supervisor and the sliding mode controllers, were implemented in the experimental test station. The reference values for the DC bus and SCBM voltages were set at $75 \mathrm{~V}$ and $30 \mathrm{~V}$, respectively.

The external load demand profile $P_{L}$, implemented through the programmable load, is presented in Fig. 5. This step series was selected to drive the system through different operating conditions that will be discussed in the sequel.

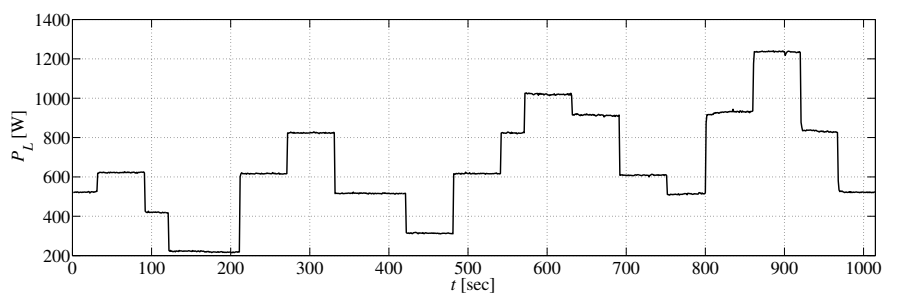

Fig. 5. External Load Demand $P_{L}$

A variable wind regime was assumed for the test. The resulting maximum available power $P_{W, \max }$ that can be obtained from the WECS module (emulated by the programmable power source) is depicted in Fig. 6.

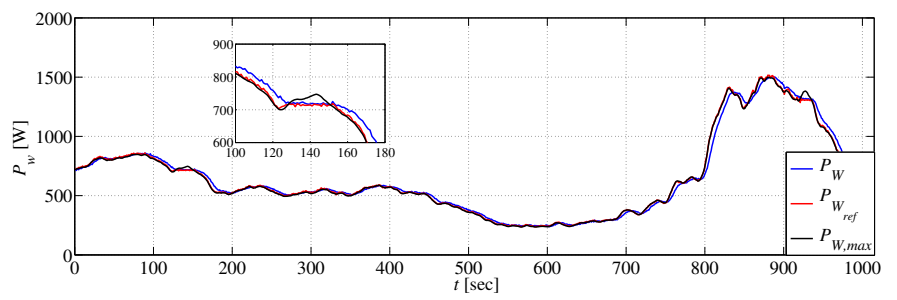

Fig. 6. Maximum available power $\left(P_{W, \max }\right)$, power reference $\left(P_{W_{r e f}}\right)$ and actual power $\left(P_{W}\right)$ of the WECS

In the same figure, the WECS power reference computed by the supervisor control, $P_{W_{\text {ref }}}$, and the actual power generated by the WECS, $P_{W}$, are shown.

Note that between 130 and $150 \mathrm{sec}$ (approximately), the maximum available wind power is higher than the total power demand $P_{t o t, r}$ (see (1)), so the WECS reference must be limitated. A similar situation occurs around $920 \mathrm{sec}$. During the rest of the time, the WECS is required to deliver its maximum available power, as shown in Fig. 6.

The evolution of the electrolyzer power $P_{E}$ (emulated by the programmable load in the tests) is displayed in Fig. 7. It can be observed that, in accordance to the supervisor control, at times when there is sufficient wind power, the electrolyzer is active. This means that the external load, the electrolyzer and the SCBM power requirements can be exclusively satisfied by the WECS module. It can be noticed in spite of the wind excess power $P_{W, b a l}$, the electrolyzer saturates at $P_{E, \max }$ (see (4)).

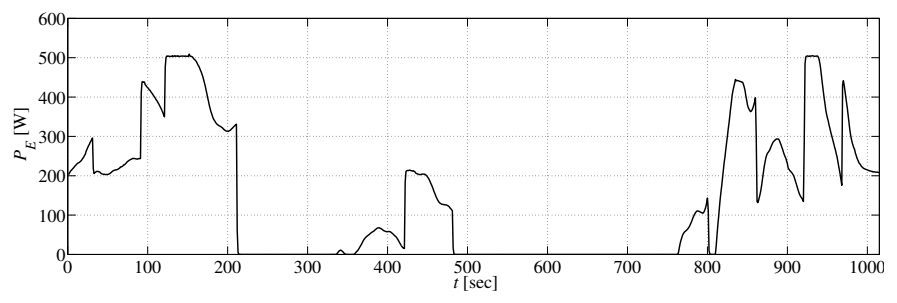

Fig. 7. Electrolyzer Power $P_{E}$

On the other hand, at times when the WECS power is not sufficient to satisfy the total power demand $P_{t o t, r}$, the FCGM module is activated (see Fig. 8). Simultaneously, the ES is turned off given that, for the sake of energy efficiency, the operations of the FCGM and of the ES are mutually exclusive (this specification can be briefly appreciated in several time periods in the presented figures).

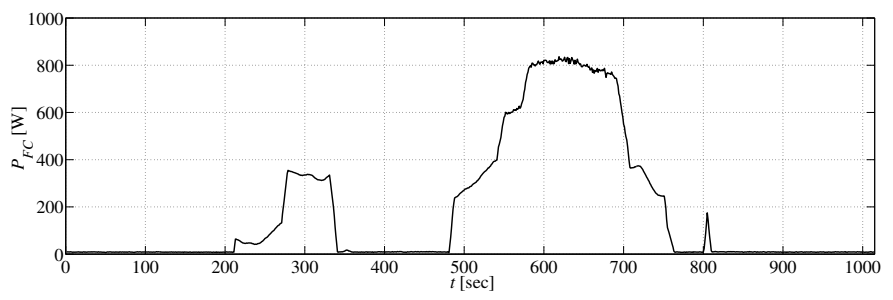

Fig. 8. FCGM Power $P_{F C}$

It is worth to stress that the FCGM current slew rate is being limited to a safe value by the sliding mode control strategy, as mentioned in Section III-B. Consequently, it is the SCBM who is responsible of regulating the DC bus voltage in the presence of abrupt load changes. It is capable to rapidly supply the load or absorb the excess power in the DC bus, complementing the slower fuel cell and WECS modules (see the $P_{S C}$ in Fig. 9). As a result, the proposed control setup efficiently maintains the DC bus voltage $v_{\text {bus }}$ within $2 \%$ of the desired value (Fig. $10)$.

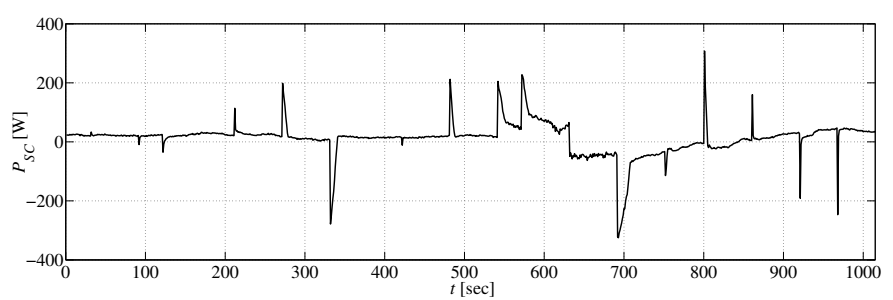

Fig. 9. SCBM Power $P_{S C}$

In Fig. 10, it also can be appreciated two zoomed areas. Those curves show in detail the controller response to abrupt load variations, obtaining a satisfactory voltage evolution. 


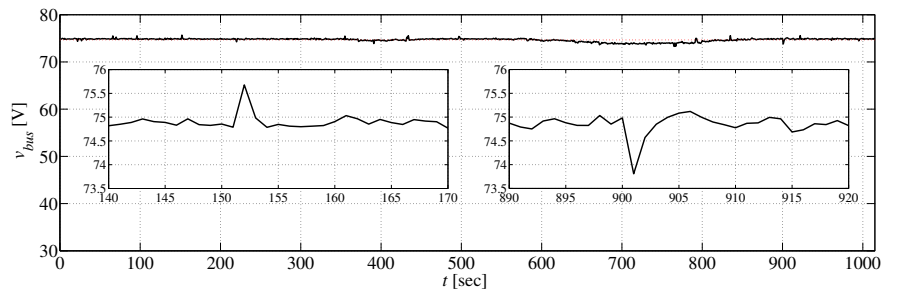

Fig. 10. DC Bus Voltage $v_{b u s}$

The relation between the FCGM voltage and current, in accordance with equation (14), can be appreciated from Fig. 11 and Fig. 12. Besides, the latter clearly displays the aforementioned slew rate limitation implemented by the FCGM sliding mode controller.

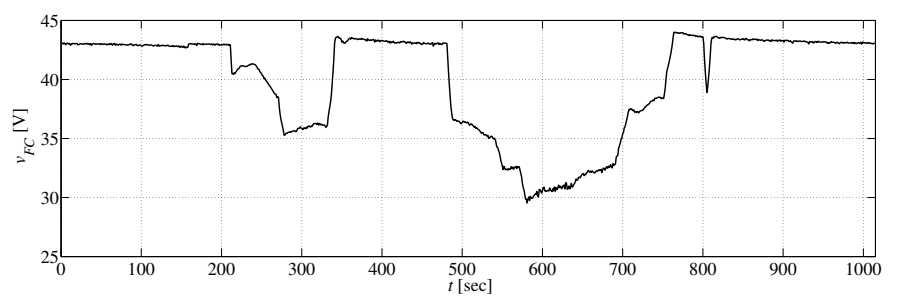

Fig. 11. FCGM Voltage $v_{F C}$

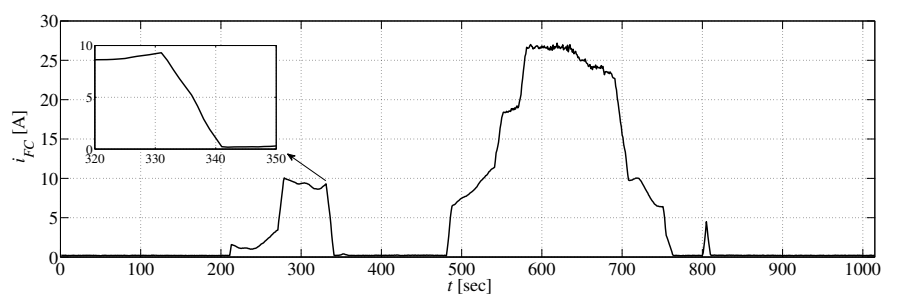

Fig. 12. FCGM Current $i_{F C}$

Finally, the SCBM voltage and current are presented in Fig. 13 and Fig. 14, respectively. It can be seen in Fig. 13 that the SCBM voltage $V_{S C}$ is maintained around the desired range of $30 \mathrm{~V}$.

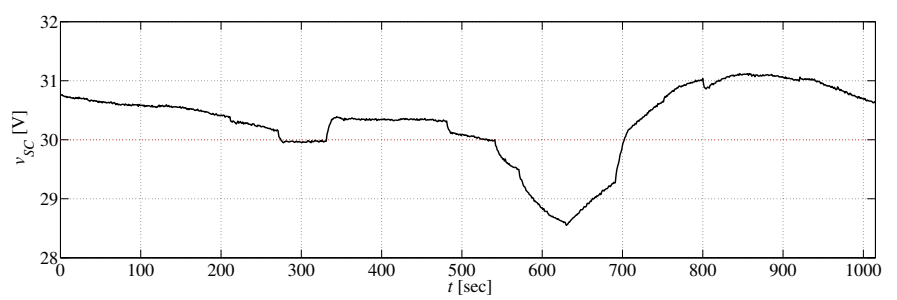

Fig. 13. SCBM $v_{S C}$

\section{CONCLUSIONS}

The theoretical design and implementation of a comprehensive control system specially intended for an autonomous fuel cell-based hybrid generation system have been thoroughly presented in this paper. Both hierarchical control levels have been introduced and analyzed in detail.

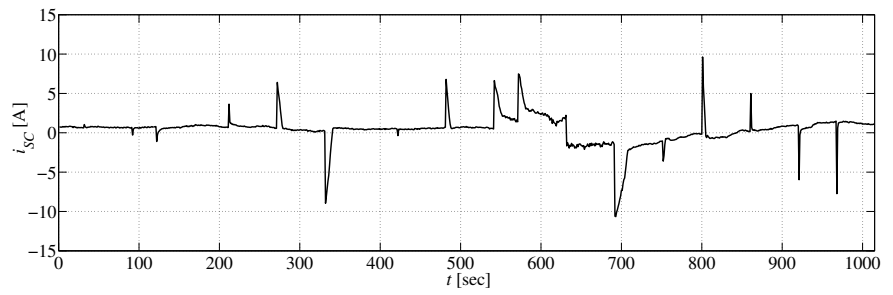

Fig. 14. SCBM Current $i_{S C}$

The proposed control setup upper level, or supervisory strategy, proved to be a proficient reference generation tool to efficiently coordinate the power interaction of the constituent subsystem, namely the fuel cell/supercapacitor-bank (main core of the HGS), the alternative energy module (emulating a WECS), the electrolyzer and the external load. For its part, the lower level constituted by dedicated sliding mode controllers for the DC-DC converters, also demonstrated its capability to robustly control the power flow and regulate the DC bus voltage, even in the presence of model uncertainties and external disturbances.

It is important to remark that, even though the two-levels control set up developed in this work is tailored to the specific HGS station available in the Institut de Robòtica $i$ Informàtica industrial (CSIC-UPC), its applicability is wideranging and can be implemented with minor modifications in many different HGS with analogous modular topologies.

Furthermore, in addition to the theoretical design guidelines, several pointers and comments have been provided, aiming to facilitate its practical realization when dealing with actual hardware and processing limitations.

Finally, it is worth to stress that, after a rigorous simulation phase, the whole control system was implemented and assessed in a test bench, under highly variable load demand and wind conditions. The successful results obtained show the suitability of the proposed control system to robustly deal with real HGS submitted to exacting operating conditions.

As future work, two main lines are being considered by the authors. Regarding the upper level, different control approaches, particularly Model Predictive Control techniques, will be explored in order to enhance the features of the proposed supervisor strategy. On the other hand, high order sliding mode controllers will be designed, implemented and assessed aiming to improve the performance of the dedicated controllers for each module.

\section{ACKNOWLEDGMENTS}

This research was supported by the Universidad Nacional de La Plata, CONICET, and SECyT, from Argentina; by FP7-PEOPLE-2011 Marie-Curie Action, Project ACRES (IIF-299767/911767) and ACOFC (PCIG09-GA2011-293876) from the EU; by Project Puma-Mind (FCHJU-2011-1-303419); partially founded by Project MICINN DPI2011-25649 and supported by the Universitat Politècnica de Catalunya, and the CSIC, from Spain. The authors would like to specially thank the proficient technical staff of the Fuel Cells Laboratory of the Institut de Robòtica i Informàtica Industrial (CSIC-UPC, Barcelona). 


\section{REFERENCES}

[1] J. Larminie and A. Dicks, Fuel Cell Systems Explained, 2nd ed. John Wiley \& Sons Inc., 2003.

[2] J. Pukrushpan, A. Stefanopoulou, and H. Peng, Control of Fuel Cell Power Systems. London: Springer, 2004.

[3] C. Kunusch, M. Mayosky, and P. Puleston, Sliding-Mode Control of PEM Fuel Cells, 1st ed. AIC - Springer., 2012.

[4] M. Nadal and F. Barbir, "Development of a hybrid fuel cell/battery powered electric vehicle," International Journal of Hydrogen Energy, vol. 21, no. 6, pp. 497 - 505, 1996. [Online]. Available: http://www.sciencedirect.com/science/article/pii/0360319995001026

[5] V. Mehta and J. S. Cooper, "Review and analysis of pem fuel cell design and manufacturing," Journal of Power Sources, vol. 114, no. 1, pp. $32-53,2003$. [Online]. Available: http://www.sciencedirect.com/science/article/pii/S0378775302005426

[6] K. Sedghisigarchi, A. Davari, and P. Famouri, "Dynamic modeling and control of a fuel cell for electric vehicle applications," in Vehicle Power and Propulsion Conference (VPPC), 2011 IEEE, sept. 2011, pp. $1-5$.

[7] J. Hamelin, K. Agbossou, A. Laperrière, F. Laurencelle, and T. Bose, "Dynamic behavior of a pem fuel cell stack for stationary applications," International Journal of Hydrogen Energy, vol. 26, no. 6, pp. 625 - 629, 2001. [Online]. Available: http://www.sciencedirect.com/science/article/pii/S036031990000121X

[8] C. Wang, M. Nehrir, and H. Gao, "Control of pem fuel cell distributed generation systems," Energy Conversion, IEEE Transactions on, vol. 21, no. 2, pp. 586 - 595, june 2006.

[9] M. Heuer, M. Kabisch, G. Heideck, and Z. Styczynski, "Pem fuel cell system as an autonomous power supplier," in Power Energy Society General Meeting, 2009. PES '09. IEEE, july 2009, pp. $1-5$

[10] F. de Bruijn and P. Veltman, "Pem fuel cells for telecom applications," in Telecommunications Energy Conference (INTELEC), 2011 IEEE 33rd International, oct. 2011, pp. $1-6$.

[11] P. Thounthong, S. Raël, and B. Davat, "Control strategy of fuel cell/supercapacitors hybrid power sources for electric vehicle," Journal of Power Sources, vol. 158, p. 806-814, 2006.

[12] P. Thounthong, V. Chunkag, P. Sethakul, B. Davat, and M. Hinaje, "Comparative study of fuel-cell vehicle hybridization with battery or supercapacitor storage device," Vehicular Technology, IEEE Transactions on, vol. 58, no. 8, pp. 3892 -3904, oct. 2009.

[13] Y. Cheng, "Assessments of energy capacity and energy losses of supercapacitors in fast charging-discharging cycles," Energy Conversion, IEEE Transactions on, vol. 25, no. 1, pp. 253 -261, march 2010.

[14] G. Saur, "Wind-to-hydrogen project: Electrolyzer capital cost study," National Renewable Energy Report (NREL) Tech Report, 2008.

[15] G. Spiazzi, P. Mattavelli, and L. Rossetto, "Sliding mode control of dc-dc converters," Cobep 97, 1997.

[16] V. Utkin, J. Gulder, and J. Shi, Sliding mode Control in ElectroMechanical Systems. London: Taylor and Francis, 1999.

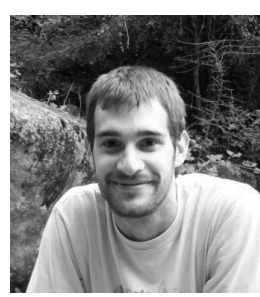

Jerónimo J. Moré received his engineer's degree in electronics (2007) and he is finishing his Ph.D. at the Universidad Nacional de La Plata (UNLP), La Plata, Buenos Aires, Argentina. He is currently Professor of the UNLP and teaching assistant at the Electrical Engineering Dept. (UNLP). He also is Assistant Researcher of the Consejo Nacional de Investigaciones Cientìficas y Técnicas (CONICET) and UNLP, Argentina. His research activities are in the area of sliding mode control of hybrid systems with applications to renewable energy systems.

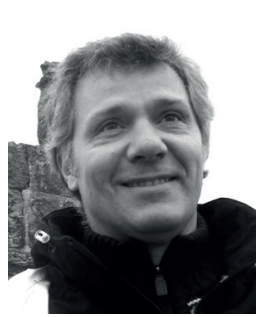

Paul Puleston received his Electronic Engineering degree (with first class Hons.) and his Ph.D. degree from the Universidad Nacional de La Plata (UNLP), Argentina, in 1988 and 1997, respectively. He is currently Full Professor at the Department of Electrical Engineering, FI-UNLP, Vice Director of the LEICI and Researcher of the Consejo Nacional de Investigaciones Científicas y Tecnicas (CONICET) at LEICI, Argentina. His main research field is automatic control systems, theory and applications, including alternative energy systems.

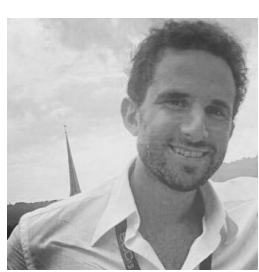

Cristian Kunusch received his B.S., MSc and $\mathrm{Ph} . \mathrm{D}$. degrees in electronic engineering from the National University of La Plata (UNLP), Argentina, in 2003, 2006 and in 2009, respectively. In 2010 , he was appointed as Postdoctoral Fellow of the Spanish National Research Council (CSIC) at the Institut de Robòtica i Informàtica Industrial (IRI) in Barcelona (Spain). Since 2014, he joined the Electric Drives Pre-Development Team of Brose Fahrzeugteile, Würzburg (Germany), as Senior Researcher. His main research interests include variable structure systems and their applications to the control and observation of fuel cell based systems and electric drives.

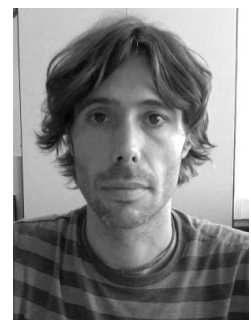

Miguel Allué Fantova was born in Huesca, Spain (1976). He received his Bachelors degrees in Industrial engineering from the University of Zaragoza and in Electronic engineering from the Polytechnic University of Catalonia (UPC), in 2001 and 2009, respectively. Since December 2001, he has been with the Control Group at the Institut de Robòtica i Informàtica Industrial (IRI) in Barcelona (Spain) where he is currently the Responsible of the "Control Laboratory of Fuel Cells and Energy systems". 